Article

\title{
An Improved Voronoi-Diagram-Based Algorithm for Continuous Facility Location Problem under Disruptions
}

\author{
Jiguang Wang *(1) and Yucai Wu \\ School of Economics \& Management, Shanxi University, Taiyuan 030006, China; wuyucai1991@email.sxu.edu.cn \\ * Correspondence: wangjg@sxu.edu.cn; Tel.: +86-139-3458-4285
}

Received: 22 July 2018; Accepted: 28 August 2018; Published: 31 August 2018

check for updates

\begin{abstract}
The classical location models implicitly assume that the facilities, once built, will always operate as planned. However, some of the facilities may become unavailable from time to time due to disruptions which highlight the urgent need to effectively manage supply chain disruptions in spite of their low probability of occurrence. Therefore, it is critical to take account of disruptions when designing a resilient supply chain network so that it performs well as a whole even after an accidental disruption. In this paper, a stylized facility location problem is considered in a continuous plane which is solved through an improved Voronoi-diagram-based algorithm under disruption risks. The research problem is to minimize the total cost in normal and failure scenarios. Furthermore, the impact of misestimating the disruption probability is also investigated. The results numerically show that although the estimated disruption probability has a significant impact on the facilities configuration, it has a minor impact on the total quantity of facilities and the expected total cost. Therefore, this paper proposes that the decision-maker should moderately overestimate disruption risk based on the "pessimistic principle". Finally, the conclusion considers managerial insights and proposes potential areas for future research.
\end{abstract}

Keywords: facility network design; disruption; continuous approximation; misestimation

\section{Introduction}

Today's turbulent, fast-changing business environment and growing complexity of globalization has imposed unexpected and inevitable risks to supply chain networks. Supply chains are susceptible to disruptions caused by various risk incidents. The consequences of disruptions are often disastrous, despite their rare occurrence. For example, Ford's fourth-quarter output in 2001 was down 13\% compared with its production plan because of a logistics disruption which was caused by airport shut down in response to the 9/11 Attacks [1]. The devastation of Toyota's parts factories in the aftermath of the 2011 tsunami in Japan caused its supply network to be disrupted and six months later, Toyota had to idle some plants in North America due to a shortage of parts, costing $\$ 72$ million in profits daily; overall auto sales in Japan fell to a 34-year low [2]. Quoting the data from Sáenz \& Revilla [3], on average, the percentage of global companies reporting a loss of revenue due to a supply chain failure increased from $28 \%$ in 2011 to $42 \%$ in 2013. In addition, according to a worldwide survey, approximately $80 \%$ of firms experienced at least one major disruption in recent years culminating in a great loss [4]. Recent global events show frequent reminders that we live in an unpredictable and changing world, and as such, many academicians and practitioners have paid a great deal of attention to supply chain facility disruptions for the purposes of enhancing supply chain decision makers' disruption risk management efficiency and effectiveness. Therefore, in such an uncertain business environment, the facility location problem has become an increasingly important topic. 
Facility location problems are among the classical Operations Research (OR) problems with a broad range of research applications [5,6]. Its essence is to make the optimal decision for facility location, capacity, route design, etc., in a given space to minimize cost or maximize market coverage. In the context of the supply chain, "Facility" usually refers to the business unit or entity which is able to systematically integrate human resources, capital, material, equipment, and technology, etc., together to produce a product or service or to achieve some planned function. Facility location decisions may be the most critical and most difficult of the decisions to accomplish an efficient and effective supply chain which aims at its strategic goal [6]. So, facility location problems have been investigated extensively in the past, due to their wide applications in various contexts.

Whereas traditional location models generally assume that facilities, once built, will always be available and perfectly reliable throughout the planning horizon. In today's complex and dynamic environment, facilities are increasingly vulnerable to partial or complete disruption which has become one of the main threats to supply chain management [7]. Various risk incidents, e.g., natural disasters, key-supplier bankruptcy, labor strikes, or terrorist attacks, showed that the business environment is becoming more and more uncertain and fragile [8-10]. Furthermore, wide spreading global procurement, decentralized production, outsourcing, supplier reduction, minimizing inventory, etc., increased the supply chain vulnerability, and highlighted the importance and urgency of proactive supply chain disruption risk management [11-13]. Therefore, the reliable facility location problem (RFLP) with the disruption risk factor considered is an important topic which needs the further investigation of scholars and practitioners.

Although supply chain management literature is now beginning to explore how to best to build resilience into supply chain network design, with increasing attention, especially toward mitigate facility disruption risks, the relational research is still in its infancy. To increase system resilience and reliability against supply chain disruptions when designing supply chain networks, some reliable models have appeared only more recently. To our knowledge, the first reliable location model was introduced by Snyder \& Daskin [14] to deal with facility disruption which started the study on the reliable location problem. Although reliable location models with disruptions consideration have gained much attention, this topic is still understudied and very few studies have focused on the continuous location problem which was introduced as an alternative for solving large-scale problems to overcome the deficiency of the discrete model $[15,16]$. Aiming to mitigate facility disruption risks by creating resilient supply chains network, this paper will consider a stylized facility location problem in a continuous plane solved through an improved Voronoi-diagram-based algorithm under the risk of disruptions. The research problem is to minimize the total system cost, including initial facility investment and expected transportation cost in normal and failure scenarios. Furthermore, the impact of misestimating the disruption probability is also investigated.

The remainder of the paper is organized as follows. The related literature is reviewed in Section 2. Section 3, states the research problem and formulates it into a continuous location problem which incorporates facility disruption risk, the empirical results are discussed. And the algorithm's solving performance is addressed by sensitivity and robustness analysis. The paper concludes in Section 4 with limitations and recommendations for future research.

\section{Literature Review}

Facility location is a well-established research area within OR. According to the decision variables, the facility location problem can be classified into discrete location problems and continuous location problems [17]. Therefore, the American Mathematical Society developed specific codes for the location problem: 90B80, discrete location and distribution; 90B85, continuous location problem [18]. The discrete location problem, usually defined as an optimal decision of the sites where new facilities are to be established, is restricted to a finite set of available candidate locations $[14,15,19]$. It seeks optimal facility locations and demand assignments to balance the tradeoff between initial fixed construction costs and day-to-day transportation costs. For example, Manthey \& Tijink [19] 
addressed the perturbation resilience by optimizing an uncapacitated discrete facility location problem. Although the discrete location model reflects reality more accurately in most cases, the researchers hold negative views on the practicality of the model in large-scale problems [20]. Most discrete location models are nondeterministic polynomial (NP) hard and suffer from excessive computational burden. To overcome this deficiency, continuum approximation (CA) models are often developed as an alternative to provide good approximate solutions to large-scale problems. CA models regard the numerous demand points can be properly approximated by a continuous function and the potential facilities are not restricted to a set of given candidate locations. Then, the whole demand plane is divided continuously and each subregion is covered by an assigned facility to satisfy it. In this way, the continuous subdemand regions and facilities could be described as a Voronoi diagram.

However, in the traditional facility location model, most of the studies assume that facilities are always available and perfectly reliable. In today's complex and dynamic environment, facilities are increasingly vulnerable to disruptions. There are numerous examples of disruptions that have resulted in significant losses to the whole supply chain network. Research on the reliable facility location problem, wherein some facilities are subject to disruption from time to time, is an emerging field in recent decade [11]. There are some different classifications about disruption scenarios in academia [21-23]. For example, the disruption originated from natural disasters, unforeseen discontinuities in supply, operational accidents, deliberate actions, information system failure, terrorism, and political instability, etc. In this paper, from the perspective of disruption inducement, the disruption scenario is divided into random disruption and nonrandom disruption. Random disruption refers to the disruptions triggered by accidental and unpredictable events, such as typhoon, earthquake, floods, fires, and other natural disasters, etc. The latter refers to the disruptions caused by deliberate actions, e.g., terrorist attacks, labor strikes, malicious competition, etc. More specifically, the disruption scenario in this study refers to the random disruption.

To increase supply chain resilience and reliability against unexpected disruptions, a number of reliable models have been proposed recently. To our best knowledge, Snyder \& Daskin [14] conducted the first research on the reliable facility location problem. With the assumption that the disruption probabilities of the facilities are mutually independent and equal, an uncapacitated fixed-charge location problem (UFLP) was studied based on a p-median problem. There are only a few papers that examine facility location problems in the presence of disruption risk using the CA model. Lim et al. [24] focused on a continuous facility location problem under disruption risk with the option of hardening selected facilities. Their model is also constructed based on the assumption that disruption probabilities are independent and the demand follows a continuous uniform distribution. Cui, Ouyang \& Shen [6] proposed a CA model to study the same problem with a relaxed assumption of homogeneous failure probabilities to location specific probabilities. Their model aims to minimize initial setup costs and expected transportation costs in normal and failure situations. Li \& Ouyang [25] expanded this direction to spatially correlated, site-dependent disruption probabilities. This paper develops a CA model to minimize the sum of initial facility construction costs and expected customer transportation costs under normal and failure scenarios. The paper also analyzes the influence of spatial distribution characteristics of continuous region and the change of disruption probability on the model. Wang \& Ouyang [26] presents game-theoretical models based on a CA scheme to optimize service facility location design under spatial competition and facility disruption risks. In addition, considering the risks of demand, supply, and finance, Jabalameli et al. [5] studied capacitated continuous location problem.

In brief, there are two limitations of the extant studies in continuous location problem. The first is the division of the target plane which is still seriously understudied. In CA models, the key issue is how to divide the whole target plane continuously as Voronoi diagram which determines the solving efficiency. To our best knowledge, in the existing related literature, Novaes et al. [27] studies the continuous facility location problem in an irregular region (Figure 1a). While the researches of Jabalameli et al. [5] (Figure 1b), Li \& Ouyang [25] (Figure 1c), and Cui, Ouyang \& Shen [6] (Figure 1d) 
transform the target area to regular geometric plane graphics. As shown in Figure $1 b-d$, the regular division on the target area aims to generalize the research. Otherwise, as shown in Figure 1a, although irregular division is more realistic, it needs to evaluate the definite integral of the six subregions. So it is still a discrete problem in essence. If the quantity of subregions increases, the solving process would become extremely difficult. Therefore, as a "compromise", the target area must be regularly divided into subregions with same geometry to achieve an expected solution. Obviously, the division rule of the target area is one of the key factors that affect the optimal solution. In addition, it should be noted, due to the low efficiency (see Figure 1b) and the complexity (see Figure 1c,d) of the division, the large-scale problems could not be addressed effectively and efficiently.

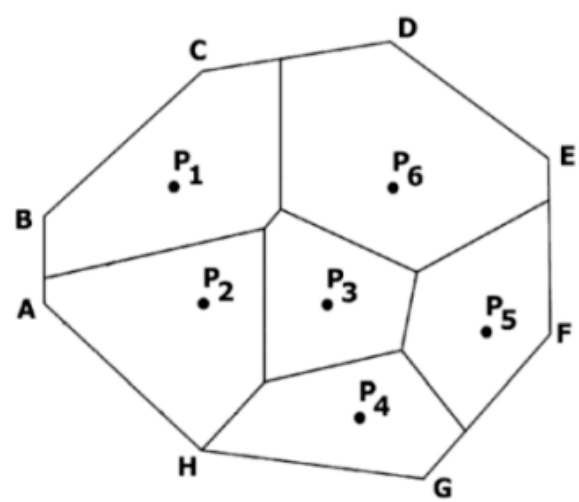

(a)

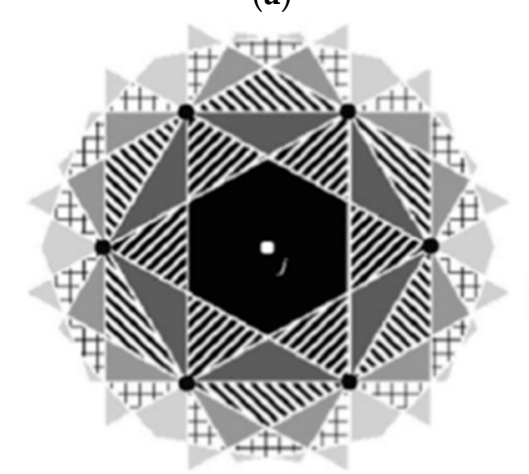

(c)

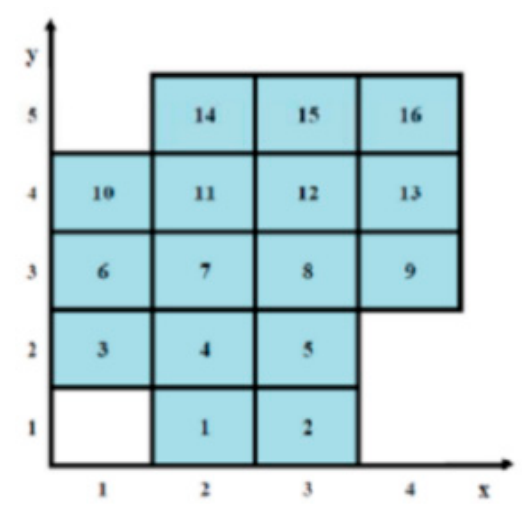

(b)

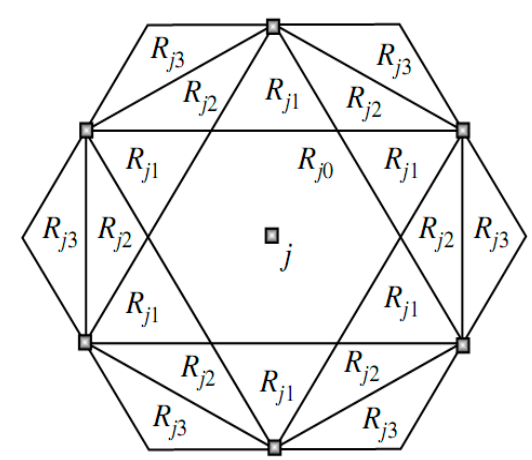

(d)

Figure 1. (a) Irregular division of target plane; (b) checker-board division of target plane; (c) quasi-regular geometric division of target plane; and (d) regular geometric division of target plane.

The second is lacking the discussion on the impact of misestimating disruption probability. The optimal solution may be changed due to the uncertainties from the market or parameter estimation error, etc. And even if all parameters are deterministic and known, the supply chain will still suffer from disruptions because of the dynamic changes of the business environment [28]. Therefore, these limitations affected the validity of the optimal solution in practical application.

To address these gaps, this paper will investigate a continuous facility location problem solved through an improved Voronoi-diagram-based algorithm under the risk of disruptions. Further, this paper will focus on the effects of the disruption probability estimation and the fault tolerance of the model.

\section{Model Formulation}

In this section, a CA model for the problem in a continuous plane is formulated which will be solved through an improved Voronoi-diagram-based algorithm under the risk of disruptions. First and foremost, how to divide a continuous plane is a key issue before constructing the CA model. This paper argues that it is the most effective and the most practical scheme to divide the target plane 
as honeycomb grid. The mathematical proofs can refer to Hales [29] and Lyon [30] on the study of "Honeycomb conjecture" which will not be discussed in this paper specifically. As a new method, a regular hexagonal grid is the best way to divide a plane into subregions of equal area and it is a significant improvement in the field of continuous location problem. Based on the assumption of the regular hexagonal grid scheme, a CA model will be formulated as follows.

Firstly, we define the relevant variables and notations which will be used throughout the paper:

$A$ : the whole demand region with area of $S$ which subjects to a uniform distribution of a density $\rho$; $q$ : the disruption probability of the supply chain facilities, $q \in(0,1)$. In this paper, due to space limitations, the disruption probability is presented as a known condition;

$c$ : the transportation cost per unit demand per unit distance;

$f_{R}$ : the setup cost of reliable facilities;

$f_{U}$ : the setup cost of unreliable facilities, $f_{R}>f_{U}$;

$r$ : the unreliable facility hardening cost factor, $r=f_{R} / f_{U}$;

$d\left(P_{1}, P_{2}\right)$ : the distance between two points, $P_{1}\left(x_{1}, y_{1}\right)$ and $P_{2}\left(x_{2}, y_{2}\right)$.

In addition, in term of the distance between two points, there are three ways of calculation usually, Euclidean distance, Manhattan distance, and Great Circle distance. Considering the specific situation of the problem, the Manhattan distance is adopted in this paper, i.e., $d\left(P_{1}, P_{2}\right)=\left|x_{1}-x_{2}\right|+\left|y_{1}-y_{2}\right|$.

The demand area $A$ is assumed uniformly distributed with a density of $\rho$. The decision maker tries to locate a set of facilities to satisfy all the demand while taking into account of random facility disruptions. Similar to Snyder \& Daskin [13], two types of facilities are considered: (1) unreliable facilities, which are subject to random disruptions; and (2) reliable facilities, which are not subject to random disruptions by additional hardened investment. In order to satisfy the whole demand region, each subregion would be served by allocating a reliable/unreliable facility as its initial allocation, where $1-q$ is the probability that the facility is available. In case the disruption of the initial allocated unreliable facilities, there is a closest reliable facility for each region as backup. In addition, the supply chain decision makers are assumed risk-neutral. Therefore, the objective of the model is to minimize the expected total $\operatorname{cost}$ (TC) of locating $n_{R}$ reliable facilities and $n_{U}$ unreliable facilities, expected transportation cost between facilities and demands.

$$
T C=f_{R} n_{R}+f_{U} n_{U}+d \cdot \rho S c
$$

where, the term $f_{R} n_{R}+f_{U} n_{U}$ is the fixed cost of locating $n_{R}$ reliable and $n_{U}$ unreliable facilities, and the term $d \cdot \rho S c$ is the expected transportation cost between facilities and demands.

Consider a subregion $G \circ$ from the whole service area $G$ that is sufficiently large. Assuming there is a possibility that the transportation cost of a nearer region to a facility maybe ignored. Thus, as shown in Figure 2a, the area of the hexagon $G \circ$ is $S \circ=\frac{3}{2} \sqrt{3} A \circ$, and the shaded part in $G \circ$ means the nearer area where the transportation cost is ignored. In addition, the Cartesian coordinate system is adopted in this paper.

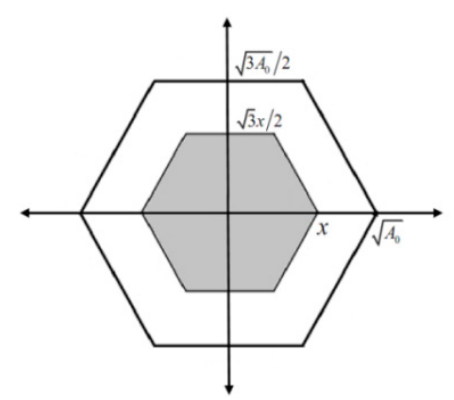

(a)

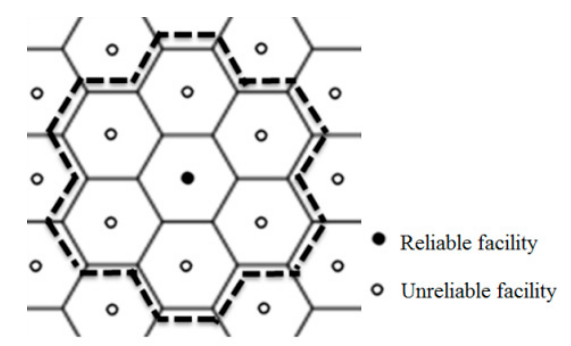

(b)

Figure 2. (a) Distance of $A \circ$ and (b) facility location set (topo-area, no $=7$ ). 
As a result, the expected distance between a facility in the center of a demand subregion and any one demand node can be obtained according to Figure 2a.

$$
\mathrm{E}[D]=\int_{G \circ} \mathrm{P}(D>x) d x=\int_{0}^{\sqrt{A \circ}}\left[1-F_{D}(x)\right] d x=\frac{2}{3} \sqrt{S \circ}
$$

where $F_{D}(x)$ is the ratio of the shaded part to the whole hexagon area, i.e., $F_{D}(x)=x^{2} /$ So.

Moreover, assuming that $G \circ$ is covered by $n \circ$ facilities and each serving a diamond area of $\mathrm{So} / \mathrm{n}$ o which means the subregion is divided further as honeycomb grid as shown in Figure $2 \mathrm{~b}$. Each subregion is a separated demand area with a deployed facility, and the $n \circ$ facilities make a set of facilities; assuming there is only one reliable facility in the facility set. Because the reliable facility does not fail, the optimal location strategy is to locate the reliable facility in the center of $A \circ$ surrounded by $n \circ-1$ unreliable facilities. Thus, each subregion will be served by the facility as its primary assignment and by the reliable facility from the center as an emergency backup assignment when one of or some of the $n \circ-1$ unreliable facilities fail.

According to formula (2), if its own facility is working in each grid, the expected distance between the facility and demand is $\frac{2}{3} \sqrt{\frac{S o}{n o}}$. Then, the expected distance between the central reliable facility and demands from its adjacent grids with unreliable facilities is represented as $\mathrm{E}\left[D_{R}\right]$. So, we know that $\frac{2}{3} \sqrt{S \circ}=\frac{2}{3} \sqrt{\frac{S \circ}{n \circ}}\left(\frac{1}{n \circ}\right)+\mathrm{E}\left[D_{R}\right]\left(\frac{n \circ-1}{n \circ}\right)$. This implies

$$
\mathrm{E}\left[D_{R}\right]=\left(\frac{n \circ}{n \circ-1}\right)\left[\frac{2}{3} \sqrt{S \circ}-\frac{2}{3 n \circ} \sqrt{\frac{S \circ}{n \circ}}\right]
$$

Furthermore, as mentioned above, considering the whole service area $G$ with total area of $S$ is composed of numerous continuously distributed subregions Go. Obviously, it is an optimal solution for a sufficiently large area. But for a finite service region, the division would be an approximate optimal solution because of its specific boundary. The whole area is divided into $n_{T}=\left(n_{R}+n_{U}\right)$ honeycomb subregions, and there are $n_{R}$ reliable facilities and $n_{U}$ unreliable facilities totally.

In each grid, the expected distance between the facility and demands is $\frac{2}{3} \sqrt{\frac{S}{n_{T}}}$. So the expected transportation cost for serving the whole demands in the service region when all the unreliable facilities failed, i.e., the expected transportation costs for serving the whole demands by all reliable facilities, is $\frac{2}{3} \sqrt{\frac{S}{n_{T}}} \rho S c\left(\frac{n_{R}}{n_{T}}\right)$. Accordingly, the expected transportation cost for serving the whole demands in the service region $G$ is

$$
\frac{2}{3}(1-q) \sqrt{\frac{S}{n_{T}}} \rho S c\left(\frac{n_{U}}{n_{T}}\right)+q \mathrm{E}\left[D_{R}\right] \rho S c\left(\frac{n_{U}}{n_{T}}\right)
$$

Furthermore, considering the proportion of unreliable facilities and reliable facilities $n_{U} / n_{R}$, each grid with reliable facility is surrounded by $n_{U} / n_{R}$ grids with unreliable facilities.

Set $A \circ=\frac{A}{n_{R}}, n \circ=\frac{n_{U}}{n_{R}}+1=\frac{n_{U}+n_{R}}{n_{R}}=\frac{n_{T}}{n_{R}}$. Therefore, $\mathrm{E}\left[D_{R}\right]$ is derived from formula (3):

$$
\mathrm{E}\left[D_{R}\right]=\frac{2}{3} \sqrt{\frac{A}{n_{R}}}\left(\frac{n_{T}}{n_{U}}\right)-\frac{2}{3} \sqrt{\frac{A}{n_{T}}}\left(\frac{n_{R}}{n_{U}}\right)
$$

Further, the expected total cost can be derived by the combination of facility setup cost and the expected transportation cost:

$$
\mathrm{E}[T C]=f_{R} n_{R}+f_{U} n_{U}+\frac{2}{3} \sqrt{\frac{A}{n_{T}}} \rho A c\left(\frac{n_{R}}{n_{T}}\right)+\frac{2}{3}(1-q) \sqrt{\frac{A}{n_{T}}} \rho A c\left(\frac{n_{U}}{n_{T}}\right)+q \mathrm{E}\left[D_{R}\right] \rho A c\left(\frac{n_{U}}{n_{T}}\right)
$$


Thus, the following is obtained by necessary algebra means:

$$
\mathrm{E}[T C]=f_{R} n_{R}+f_{U} n_{U}+q \psi \sqrt{\frac{1}{n_{R}}}+(1-q) \psi \sqrt{\frac{1}{n_{T}}}
$$

where $\psi=\frac{2}{3} \rho A^{\frac{3}{2}} c$ is a constant.

The optimal quantity of reliable and unreliable facilities can be obtained by taking the derivative of formula (7):

$$
n_{R}^{*}=\left(\frac{\psi q}{2\left(f_{R}-f_{U}\right)}\right)^{\frac{2}{3}}, n_{U}^{*}=\left(\frac{\psi(1-q)}{2 f_{U}}\right)^{\frac{2}{3}}-\left(\frac{\psi q}{2\left(f_{R}-f_{U}\right)}\right)^{\frac{2}{3}}
$$

Then, $n_{T}^{*}=n_{R}^{*}+n_{U}^{*}=\left(\frac{\psi(1-q)}{2 f_{U}}\right)^{\frac{2}{3}}$.

Since $n_{R}^{*} \leq n_{T}^{*}$, the optimal solution is given as follows:

When $q \leq 1-f_{U} / f_{R}$, the optimal solution is formula (8); when $q>1-f_{U} / f_{R}$, it is optimal to deploy only reliable facilities or harden all unreliable facilities to reliable ones as shown in formula (9),

$$
n_{R}^{*}=\left(\frac{\psi}{2 f_{R}}\right)^{\frac{2}{3}}, n_{U}^{*}=0
$$

Therefore, the optimal solution of the problem is given by:

Proposition 1. The disruption probability threshold $q_{t h}=1-f_{U} / f_{R}$ divides supply chain network into two categories:

When $q \leq 1-f_{U} / f_{R}$, it is optimal to deploy both two types of facilities as formula (8) stated;

When $q>1-f_{U} / f_{R}$, it is optimal to deploy only reliable facilities or harden all unreliable facilities to reliable facilities as formula (9) stated.

Based on Proposition 1, the threshold $q_{t h}$ approaches zero when $f_{R}$ is close to $f_{U}$. This means that if the disruption probability of supply chain facilities is large or the incremental hardening cost of unreliable facilities is relatively small, the best decision is to deploy only reliable facilities. On the contrary, if the disruption probability is relatively small or the hardening cost of unreliable facilities is large enough, it is optimal to deploy both two types of facilities.

It should be noted that the precondition of this paper is that all the demands should be fully met, which means the supply chain network as a whole is not disrupted in virtue of the reliable facilities. Therefore, considering all facilities are unreliable is outside of the scope of this paper.

By substituting formulas (8) and (9) into formula (7), the optimal expected total cost $\mathrm{E}\left[\mathrm{TC}\left(n_{R}^{*}, n_{U}^{*}\right)\right]$ is obtained:

$$
\mathrm{E}\left[\mathrm{TC}\left(n_{R}^{*}, n_{U}^{*}\right)\right]= \begin{cases}3\left(\frac{\psi}{2}\right)^{\frac{2}{3}}\left[q^{\frac{2}{3}}\left(f_{R}-f_{U}\right)^{\frac{1}{3}}+(1-q)^{\frac{2}{3}} f_{U}^{\frac{1}{3}}\right], & q \leq q_{t h} \\ 3\left(\frac{\psi}{2}\right)^{\frac{2}{3}} f_{R^{\prime}}^{\frac{1}{3}}, & q>q_{t h}\end{cases}
$$

In this section, the numerical experiments will be used to show the advantage of the CA model for the problem in a continuous plane. Assuming that the target demand area $A$ is $150,000 \mathrm{~km}^{2}$ which is uniformly distributed with a density of $\rho=300$; setup cost of reliable and unreliable facilities are $f_{R}=1,500,000, f_{U}=1,000,000$; the transportation cost per unit demand per unit distance $c=0.75$; the facilities disruption probability $q=0.05$.

Therefore, the optimal solution can be obtained (Table 1). 
Table 1. Optimal solution.

\begin{tabular}{|c|c|c|c|c|c|}
\hline$\left(f_{R}, f_{U}\right)$ & $r$ & & $\begin{array}{l}\text { Reliable } \\
\text { Facilities }\end{array}$ & Unreliable Facilities & Total \\
\hline$\left(1.5 \times 10^{6}, 1 \times 10^{6}\right)$ & 1.5 & $\begin{array}{l}\text { Facility quantity } \\
\text { Facility cost } \\
\text { Transportation cost } \\
\text { Subtotal cost } \\
\text { Total cost }\end{array}$ & $\begin{array}{c}10 \\
1.5 \times 10^{7} \\
\text { Initial allocation } \\
2.069 \times 10^{7} \\
3.569 \times 10^{7}\end{array}$ & $\begin{array}{c}34 \\
3.4 \times 10^{7} \\
\text { Backup allocation } \\
7.730 \times 10^{7} \\
11.130 \times 10^{7} \\
14.699 \times 10^{7}\end{array}$ & $\begin{array}{c}44 \\
4.9 \times 10^{7} \\
9.799 \times 10^{7}\end{array}$ \\
\hline
\end{tabular}

In supply chain literature on reliable facility location, the estimation of disruption probability is usually assumed perfectly accurate while there has very limited discussion on the misestimating disruption probability [24,31,32]. However, as mentioned above, the uncertainty in dynamic environments or misestimating the disruption probability would definitely affect the optimal solution. Therefore, this paper argues that it is necessary to further analyze the impact of misestimating the disruption probability to enhance the robustness of the model.

Firstly, the sensitivity of the optimal solution $n_{R}^{*}, n_{T}^{*}$ to the disruption probability $q$ is analyzed.

Based on formula (8), $n_{R}^{*}=\left(\frac{\psi q}{2\left(f_{R}-f_{U}\right)}\right)^{\frac{2}{3}}, n_{T}^{*}=\left(\frac{\psi(1-q)}{2 f_{U}}\right)^{\frac{2}{3}}$, we obtained the derivations of $q$ : $\frac{d n_{R}^{*}}{d q}=\frac{2}{3}\left(\frac{\psi}{2\left(f_{R}-f_{U}\right)}\right)^{\frac{2}{3}} q^{-\frac{1}{3}}, \frac{d n_{T}^{*}}{d q}=-\frac{2}{3}\left(\frac{\psi}{2 f_{U}}\right)^{\frac{2}{3}}(1-q)^{-\frac{1}{3}}$.

Then the sensitivity analysis of the numerical example is showed in Table 2.

Table 2. Sensitivity analysis of $n_{R}^{*}, n_{T}^{*}$ to $q$.

\begin{tabular}{cccccccc}
\hline & $\boldsymbol{q}=\mathbf{0 . 0 1}$ & $\boldsymbol{q}=\mathbf{0 . 0 5}$ & $\boldsymbol{q}=\mathbf{0 . 1}$ & $\boldsymbol{q}=\mathbf{0 . 1 5}$ & $\boldsymbol{q}=\mathbf{0 . 2}$ & $\boldsymbol{q}=\mathbf{0 . 3}$ & $\boldsymbol{q}=\mathbf{0 . 4}$ \\
\hline$\frac{d n_{R}^{*}}{d q}$ & 482.75 & 131.04 & 104.00 & 90.86 & 82.55 & 72.12 & 65.60 \\
$\frac{d n_{T}^{*}}{d q}$ & -30.42 & -30.94 & -31.50 & -32.10 & -32.76 & -34.23 & -36.05 \\
\hline
\end{tabular}

The result shows that when $q$ is small (e.g., $q \in(0,0.15)), n_{R}^{*}$ is relatively sensitive to $q$; while $n_{T}^{*}$ is not sensitive to $q$. So, when the $q$ is small, the total quantity of facilities is relatively stable and the quantity of reliable facilities will vary significantly with $q$.

Then is the further study on the impact of key parameters on optimal solution. This paper will investigate how the disruption probability $q$ and the facility hardening cost factor $r$ affect the expected total cost and the quantities of the two types of facilities. Firstly, assuming the value of $f_{U}$ and $c$ remains fixed at its initial value, and the value of $q$ is supposed to change from 0.01 to 0.5 . Then, in addition to initial $f_{R}=1,500,000(r=1.5)$, we will consider the following three situations, i.e., $f_{R}=1,250,000$, $f_{R}=1,750,000$, and $f_{R}=2,000,000$, accordingly, we obtain the value of $r$ in four situations, i.e., 1.25, 1.5, 1.75 , and 2, respectively. The results are shown in Figure 3a,b.

Figure 3a illustrates the changes of the expected total cost with $q$ and $r$, and Figure $3 \mathrm{~b}$ reflects the relation of the facility quantities with $q$ and $r$. Obviously, the quantity of facilities is relatively sensitive to $q$ when $q$ is small, and when $q$ is large, all facilities are hardened and upgraded to reliable ones.

In addition, as Figure $3 b$ shows, it is interesting to note that both facilities will be deployed when the disruption probability is lower than the threshold $q_{t h}$. However, when the disruption probability is larger than the threshold $q_{t h}$, only the reliable facilities will be deployed. This suggests that misestimation will significantly impact the supply chain network design, especially when the disruption probability is small. Therefore, in the next section, how the misestimation of the disruption probability impacts the expected total cost will be addressed. 


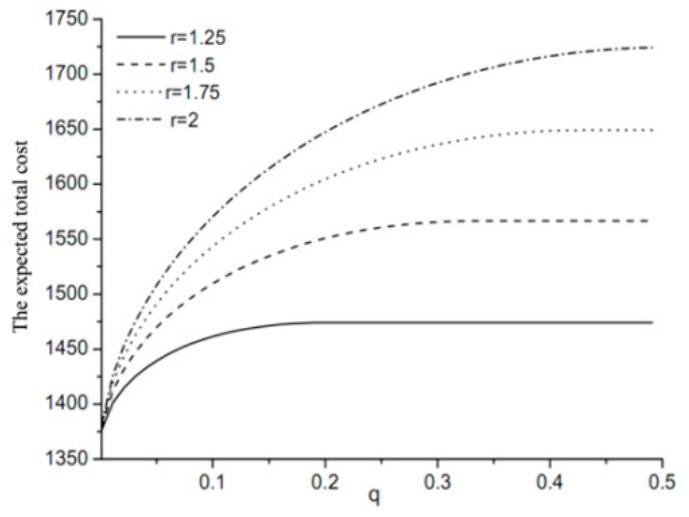

(a)

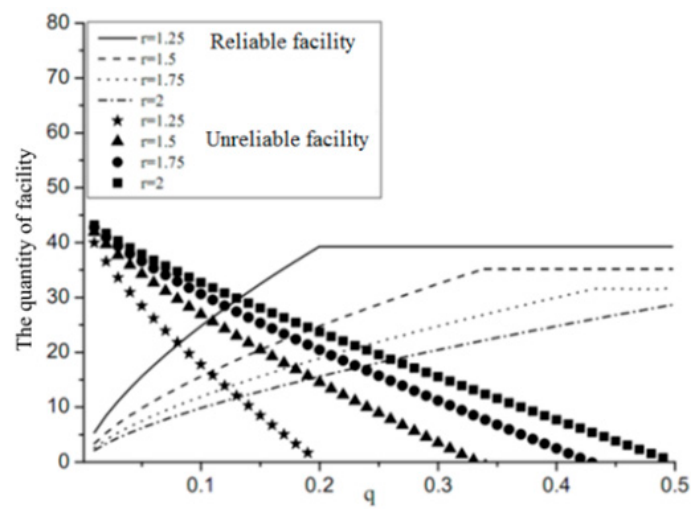

(b)

Figure 3. (a) Sensitivity analysis of expected total cost to $q \& r$ and (b) sensitivity analysis of optimal facility quantity to $q \& r$.

The second is the robustness analysis on the model to investigate the impact of misestimating the disruption probability. Due to the sparseness of historical data on disruptions and the emerging of new types of disruptions, it may be very difficult to estimate the disruption probability accurately in practice. Hence, it is important to consider the robustness of the model. Robustness analysis is applicable when uncertainty is a factor of problem situations. In addition, the adaptability and fault-tolerant capability of the model also would be discussed by robustness analysis to explore the practicability of the model.

Furthermore, since the misestimation of disruption probability impacts the expected total cost, the absolute and relative changes of the expected total cost when $r=1.5$ (Figure 4a,b) will be investigated first. In both Figure $4 a, b, q$ is the true disruption probability, $\hat{q}$ is the estimated disruption probability, $\hat{q}-q$ is the estimation error, and $(\hat{q}-q) / q \times 100 \%$ is the estimation error rate of $q$ respectively.

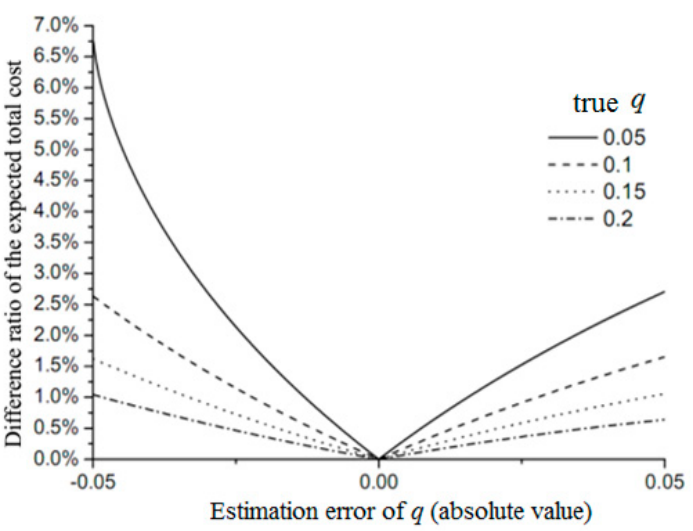

(a)

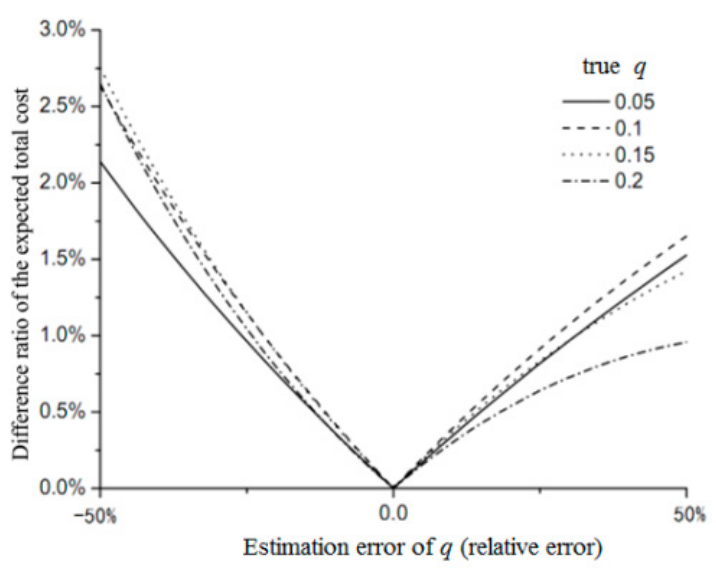

(b)

Figure 4. (a) The estimation error of $q$ and the difference ratio of the expected total cost (absolute error).

(b) The estimation error of $q$ and the difference ratio of the expected total cost (relative error).

Comparing Figure $4 \mathrm{a}, \mathrm{b}$, although the misestimation will significantly impact the supply chain network design, the expected total cost is not sensitive to the estimation error of $q$. For example, when the true disruption probability $q=0.05$ and the estimated disruption probability $\hat{q}=0.001$ which has dramatically changed the quantity of facilities, the impact on the expected total cost is less than $7 \%$. Even when relative estimation error is $50 \%$, the impact on the expected total cost is still less than $3 \%$. 
Furthermore, the different combinations of $q$ and $\hat{q}$ are presented in Table 3 in which the expected total cost will significantly change with the increase of $r$.

Table 3. The different ratio of expected total cost caused by estimation error of $q(\%)$.

\begin{tabular}{lcccccccccc}
\hline & True $\boldsymbol{q}$ & $\mathbf{0 . 0 1}$ & $\mathbf{0 . 0 5}$ & & $\mathbf{0 . 1}$ & & $\mathbf{0 . 1 5}$ & $\mathbf{0 . 2}$ \\
\cline { 2 - 9 } & Estimated $\boldsymbol{q}$ & $\mathbf{0 . 0 5}$ & $\mathbf{0 . 0 1}$ & $\mathbf{0 . 1}$ & $\mathbf{0 . 0 5}$ & $\mathbf{0 . 1 5}$ & $\mathbf{0 . 2}$ & $\mathbf{0 . 1}$ & $\mathbf{0 . 2}$ & $\mathbf{0 . 1}$ \\
\hline \multirow{2}{*}{ Difference Ratio of Expected Total Cost } & $r=1.25$ & 2.87 & 2.79 & 1.52 & 1.5 & 0.68 & 0.87 & 0.68 & 0.19 & 0.87 \\
& $r=1.5$ & 4.27 & 4.09 & 2.71 & 2.63 & 1.65 & 2.72 & 1.62 & 1.05 & 2.65 \\
& $r=2$ & 6.00 & 5.66 & 4.13 & 3.97 & 2.79 & 4.89 & 2.71 & 2.05 & 4.67 \\
& $r=3$ & 8.14 & 7.52 & 5.82 & 5.50 & 4.10 & 7.40 & 3.94 & 3.16 & 6.89 \\
\hline
\end{tabular}

The above analysis shows that, although the estimated disruption probability has a significant impact on the facilities deployment, the impact on the total quantity of facilities and the expected total cost is very small which means the model has strong robustness. Further, according to Figure $4 a$, the impact of misestimation of $q$ is relatively large when the value of $q$ is small. But the impact will be reduced with the increase of $q$. When $q>q_{t h}$, e.g., $q_{t h}=0.2$, the changes of $q$ will not affect the total cost because all the facilities have been upgraded to reliable facilities.

In addition, although the studies above show that the model is relatively robust to the misestimation of $q$, the misestimation interval will increase when the disruption probability increase. Therefore, it is particularly important to consider the solving performance of the model in the worst case scenario. As shown in Figure 5, the expected total cost error will be less than $12 \%$ when $r=1.25$ and the misestimation of disruption probability is $\hat{q} \in[0,0.5]$. The bottom diagonal means no misestimation error.

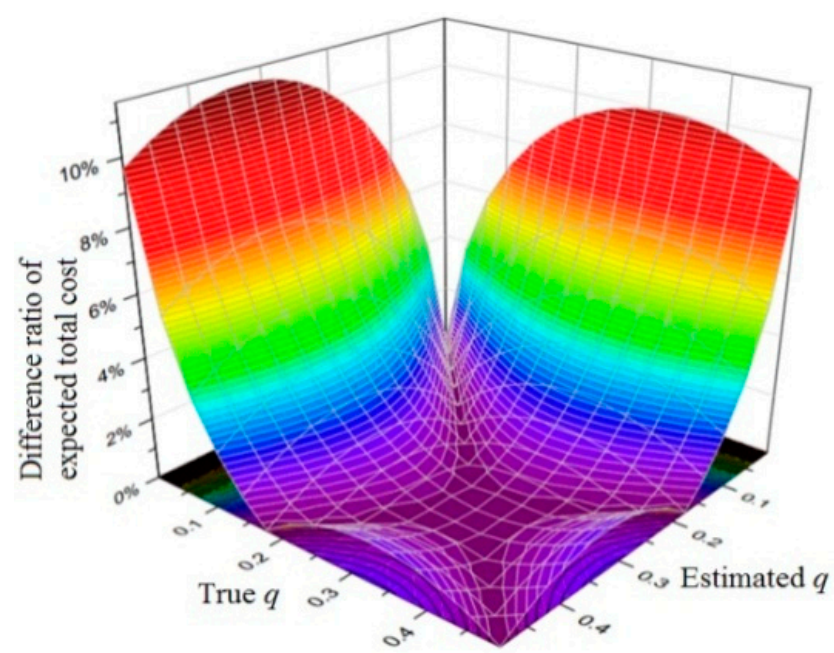

Figure 5. The misestimation of disruption probability $q$ and the difference ratio of expected total cost.

In addition, by comparing the Figures 4 and 5, this paper finds that the expected total cost difference caused by underestimation of the disruption probability $q$ is significantly larger than that of the equivalent overestimation. Therefore, the finding supports the view of this paper: the decision maker should moderately overestimate the disruption risk of the supply chain facilities based on the "pessimistic principle". Although the quantity of reliable facilities will increase in a certain degree, the total quantity of facilities is relatively stable.

\section{Conclusions}

This paper considers a facility location problem in the presence of random facility disruption with imperfect estimation of the disruption risk. This paper proposes a new research program that divides 
the target area according to scientific honeycombed division, and the continuous approximation model is constructed to deal with the plane continuous facility location problem. Further, this paper discussed the algorithm's solving performance by sensitivity analysis and robustness analysis in consideration of the imperfect estimation on the disruption risk.

Theoretically, this study contributes to the literature in a number of ways. First of all, this study adds to the emerging literature on supply chain sustainability community from an operational dimension. As discussed above, the failure of the supply chain would incur actual negative impacts on operational continuity and sometimes even threaten the very survival of the firms involved in the supply chain. This paper provides a new research program theoretically. Second, this paper improved the performance of the Voronoi-diagram-based algorithm. As discussed above, a regular hexagonal grid is the best way to divide a plane into subregions of equal area and it is a significant improvement in the field of continuous location problem. Third, the supply chain literature has very limited discussion on the impact of misestimating disruption probability. This study fills this gap by comprehensively analyzing the robustness of the model.

Managerially, based on our findings, some managerial insights and guidelines are summarized for decision makers designing a resilient supply chain network. (1) When the disruption probability $q$ is large enough, the supply chain decision makers should upgrade all the facilities to the reliable one. On the contrary, when the disruption probability is small, two types of facilities are deployed. So, the disruption probability threshold $q_{t h}$ is obtained. It shows that $q_{t h}$ is only related to the cost of two types of facilities, rather than the disruption probability $q$. (2) When the true disruption probability is relatively small, the expected total cost is not sensitive to the changes of disruption probability $q$. Therefore, misestimation to a certain degree is equally acceptable. More specifically, only if the unreliable facility hardening cost factor $r$ and estimation error of disruption probability is not too large, the misestimation of disruption probability just slightly affects the expected total cost. Therefore, the validity of the model is further proved. (3) The expected total cost difference caused by the underestimation of disruption probability $q$ is significantly higher than that of the equivalent overestimation. It means the decision-makers should moderately overestimate the risk of disruption based on the "pessimistic principle". Therefore, taking all the demands is fully met as a prerequisite, i.e., the supply chain network undisrupted as a whole, although overestimation of disruption probability will increase the quantity of reliable facilities, to a certain degree, the total quantity of facilities is relatively stable. This principle is more practicable in actual decision-making.

A limitation of this study poses new questions and promising future research. First, this paper does not consider partial facility failures that make a facility partially reliable. Second, the disruption probability of unreliable facilities is considered as random and independent in this paper. Last, capacity is not considered as a constraint variable in this paper based on the classical UFLP. It would be informative to investigate how insights would change given capacity as an endogenous variable. So, to implement these works in future, the model would be redefined by introducing new decision variables, and the algorithm has to be modified both.

Author Contributions: J.W. contributed to the whole process of research and manuscript writing; Y.W. contributed to the whole process of research and manuscript writing. Both authors have approved the final article.

Funding: This research was funded by the MOE (Ministry of Education in China) Project of Humanities and Social Sciences (grant numbers 16YJC630116).

Conflicts of Interest: The authors declare no conflicts of interest. The funders had no role in the design of the study; in the collection, analyses, or interpretation of data; in the writing of the manuscript, and in the decision to publish the results.

\section{References}

1. Rice, J.B.; Sheffi, Y. A supply chain view of the resilient enterprise. MIT Sloan Manag. Rev. 2005, 47, 41-48.

2. Pettit, T.J.; Croxton, K.L.; Fiksel, J. Ensuring Supply Chain Resilience: Development and Implementation of an Assessment Tool. J. Bus. Log. 2013, 34, 46-76. [CrossRef] 
3. Sáenz, M.J.; Revilla, E. Creating more resilient supply chains. MIT Sloan Manag. Rev. 2014, 55, $22-24$.

4. Business Continuity Institute (BCI). Supply Chain Resilience Report. 2017. Available online: https://www. thebci.org/resource/supply-chain-resilience-report-2017.html (accessed on 6 May 2017).

5. Jabalameli, M.S.; Hosseininezhad, S.J.; Jalali Naini, S.G. A risk management model for the plant location problem. Int. J. Manag. Sci. Eng. Manag. 2013, 8, 93-101.

6. Cui, T.; Ouyang, Y.; Shen, Z.M. Reliable Facility Location Design under the Risk of Disruptions. Oper. Res. 2010, 58, 998-1011. [CrossRef]

7. Li, R.; Dong, Q.; Jin, C.; Kang, R. A New Resilience Measure for Supply Chain Networks. Sustainability 2017, 9, 144. [CrossRef]

8. Tomlin, B. On the Value of Mitigation and Contingency Strategies for Managing Supply Chain Disruption Risks. Manag. Sci. 2006, 52, 639-657. [CrossRef]

9. Zegordi, S.H.; Davarzani, H. Developing a supply chain disruption analysis model: Application of colored Petri-nets. Expert Syst. Appl. 2012, 39, 2102-2111. [CrossRef]

10. Bao, S.; Zhang, C.; Ouyang, M.; Miao, L. An integrated tri-level model for enhancing the resilience of facilities against intentional attacks. Ann. Oper. Res. 2017. [CrossRef]

11. Chopra, S.; Sodhi, M.S. Reducing the Risk of Supply Chain Disruptions. MIT Sloan Manag. Rev. 2014, $55,73-80$.

12. Wagner, S.M.; Neshat, N. Assessing the vulnerability of supply chains using graph theory. Int. J. Prod. Econ. 2010, 126, 121-129. [CrossRef]

13. López, C.; Ishizaka, A. A hybrid FCM-AHP approach to predict impacts of offshore outsourcing location decisions on supply chain resilience. J. Bus. Res. 2017. [CrossRef]

14. Snyder, L.V.; Daskin, M.S. Reliability models for facility location: The expected failure cost case. Transporta Sci. 2005, 39, 400-416. [CrossRef]

15. Snyder, L.V.; Atan, Z.; Peng, P.; Rong, Y.; Schmitt, A.J.; Sinsoysal, B. OR/MS Models for Supply Chain Disruptions: A Review. IIE Trans. 2016, 48, 89-109. [CrossRef]

16. Sawik, T. On the fair optimization of cost and customer service level in a supply chain under disruption risks. Omega 2015, 53, 58-66. [CrossRef]

17. Melo, M.T.; Nickel, S.; Saldanhadagama, F. Facility location and supply chain management-A review. Eur. J. Oper. Res. 2009, 196, 401-412. [CrossRef]

18. MSC. Mathematics Subject Classification (MSC), 2000. Available online: http://www.emis.de/MSC2000/ (accessed on 18 March 2017).

19. Manthey, B.; Tijink, M.B. Perturbation resilience for the facility location problem. Oper. Res. Lett. 2018, 46, 215-218. [CrossRef]

20. Basu, S.; Sharma, M.; Ghosh, P.S. Metaheuristic applications on discrete facility location problems: A survey. Opsearch 2015, 52, 530-561. [CrossRef]

21. Chen, K.; Xiao, T. Demand disruption and coordination of the supply chain with a dominant retailer. Eur. J. Oper. Res. 2009, 197, 225-234. [CrossRef]

22. Tang, C.S. Robust strategies for mitigating supply chain disruptions. Int. J. Log.-Res. Appl. 2006, 9, $33-45$. [CrossRef]

23. Kleindorfer, P.R.; Saad, G.H. Managing Disruption Risks in Supply Chains. Prod. Oper. Manag. 2009, 14, 53-68. [CrossRef]

24. Lim, M.K.; Bassamboo, A.; Chopra, S.; Daskin, M.S. Facility Location Decisions with Random Disruptions and Imperfect Estimation. Manuf. Serv. Oper. Manag. 2013, 15, 239-249. [CrossRef]

25. Li, X.; Ouyang, Y. A Continuum Approximation Approach to Reliable Facility Location Design under Correlated Probabilistic Disruptions. Transp. Res. B-Meth. 2010, 44, 535-548. [CrossRef]

26. Wang, X.; Ouyang, Y. A continuum approximation approach to competitive facility location design under facility disruption risks. Transp. Res. B-Meth. 2013, 50, 90-103. [CrossRef]

27. Novaes, A.G.; De Cursi, J.E.; Silva, A.C.; Souza, J.C. Solving continuous location-districting problems with Voronoi diagrams. Comput. Oper. Res. 2009, 36, 40-59. [CrossRef]

28. Shu, T.; Gao, X.; Chen, S.; Wang, S.; Lai, K.K.; Gan, L. Weighing efficiency-robustness in supply chain disruption by multi-objective firefly algorithm. Sustainability 2016, 8, 250. [CrossRef]

29. Hales, T.C. The Honeycomb Conjecture. Discrete Comput. Geom. 2001, 25, 1-22. [CrossRef] 
30. Lyon, A. Mathematical Explanations of Empirical Facts, And Mathematical Realism. Austral J. Philos. 2012, 90, 559-578. [CrossRef]

31. Yun, L.; Qin, Y.; Fan, H.; Ji, C.; Li, X.; Jia, L. A reliability model for facility location design under imperfect information. Transp. Res. B-Meth. 2015, 81, 596-615. [CrossRef]

32. Berman, O.; Krass, D.; Menezes, M.B. Locating Facilities in the Presence of Disruptions and Incomplete Information. Decis. Sci. 2009, 40, 845-868. [CrossRef] 